\title{
Local Cutaneous Effects Associated with Chlorhexidine-Impregnated Gel Dressing in Hematopoietic Stem Cell Transplantation Patients
}

\author{
Bruna Nogueira dos Santos ${ }^{1}$, Maria Carolina de Oliveira ${ }^{2}$, \\ Fernanda Titareli Merizio Martins Braga1, Amanda Salles Margatho3, \\ Laís Carvalho Castanho Esparrachiari' ${ }^{1}$, Renata Cristina de Campos Pereira Silveira1 \\ ${ }^{1}$ Department of General and Specialized Nursing, Ribeirão Preto College of Nursing, University of São Paulo, Ribeirão Preto, \\ Brazil \\ ${ }^{2}$ Division of Clinical Immunology, Ribeirão Preto Medical School, University of São Paulo, Ribeirão Preto, Brazil \\ ${ }^{3}$ Department of Nursing, State University of Londrina, Londrina, Brazil \\ Email: brunandsantos@hotmail.com
}

How to cite this paper: dos Santos, B.N., de Oliveira, M.C., Braga, F.T.M.M., Margatho, A.S., Esparrachiari, L.C.C. and de Campos Pereira Silveira, R.C. (2018) Local Cutaneous Effects Associated with ChlorhexidineImpregnated Gel Dressing in Hematopoietic Stem Cell Transplantation Patients. Open Journal of Nursing, 8, 115-129. https://doi.org/10.4236/ojn.2018.82010

Received: January 4, 2018

Accepted: February 10, 2018

Published: February 13, 2018

Copyright $\odot 2018$ by authors and Scientific Research Publishing Inc. This work is licensed under the Creative Commons Attribution International License (CC BY 4.0).

http://creativecommons.org/licenses/by/4.0/

\section{(c) (i) Open Access}

\begin{abstract}
Introduction: Hematopoietic stem cell transplantation (HSCT) often requires a central venous catheter (CVC) for quick and safe vascular access. Currently, new technologies are available to protect the catheter insertion site, such as chlorhexidine-impregnated gel dressings (CIGD). Objectives: To evaluate local cutaneous effects associated with CIGD in patients undergoing HSCT. Methods: In this cross-sectional, prospective study, we evaluated 25 HSCT patients who had a CVC inserted. Patients were visited daily to monitor the CIGD changing procedures and evaluate abnormalities of the underlying skin after dressing removal. Findings: Local erythema was the most frequently detected abnormality, although usually transient and considered secondary to the mechanical trauma of dressing removal. The most severe lesions, consisting of areas of skin loss, erythematous plaques and/or vesicles were classified as skin irritation and presented in 11 (44\%) of the 25 patients. An association test showed that skin irritation was more frequent in patients who underwent allogeneic HSCT ( $p=0.03$ ). Skin irritation was most frequently observed in areas of contact with the non-woven polyester adhesive tape $(n=22 ; 88 \%)$, which made up the adhesive margins of the dressing. The CIGD was discontinued in 6 (54\%) of the 11 patients who presented severe skin injuries. Conclusion: In this study, we detected that skin irritation was more frequent in patients who underwent allogeneic HSCT. The most common skin manifestation was skin loss.
\end{abstract}




\section{Keywords}

Bandages, Catheterization, Central Venous Catheters, Hematopoietic Stem

Cell Transplantation, Skin Abnormalities

\section{Introduction}

Hematopoietic Stem Cell Transplantation (HSCT) refers to a procedure where normal hematopoietic stem cells are given to a recipient to reconstitute hematopoiesis. It is a potentially curative therapy for many life-threatening cancers, and onco-hematologic, genetic, and autoimmune diseases [1] [2] [3]. HSCT using the patient's own hematopoietic cells is called autologous HSCT, and when the hematopoietic cells derive from a donor (family members, volunteer donors, or umbilical cord blood banks), it is called allogeneic HSCT. Stem cells can be derived from bone marrow, peripheral blood, or umbilical cord blood [1] [2] [3]. Before infusion of stem cells, patients are treated with a conditioning regimen, which may include high dose chemotherapy, immunotherapy and/or radiotherapy, aiming to decrease the function of the hematopoietic system and treat the underlying disease [2]. Central Venous Catheters (CVC) are commonly used in patients with hematological malignancies to enable HSCT therapy [4] as they are useful for infusion of drugs, including chemotherapy; infusion of blood products and total parenteral nutrition; blood withdrawal; and hemodynamic monitoring [4] [5] [6]. There are two different types of long-term CVC used for prolonged treatments (more than 14 days) in HSCT: surgically implanted tunneled CVC (e.g., Hickman catheter) with a tunneled portion exiting the skin and a cuff inside the exit site, and non-tunneled CVC which is percutaneously inserted into central veins [5]. One harmful effect caused by the use of CVC is the risk of central line-associated bloodstream infection (CLABSI). In non-tunneled CVC, this occurs through migration of microorganisms from the skin to the catheter tip, direct contamination of the catheter by hand contact or infusion of contaminated fluids [4] [7] [8]. In tunneled catheters, the hub is the major source of contamination [9]. Dressings used to protect the catheter exit site provide a protective barrier to prevent migration of skin microorganisms into the cutaneous catheter tract, as well as preventing direct contamination of the catheter through contact with hands and other materials, allowing catheter stabilization by reducing movement and preventing accidental removal or partial dislodgment of the catheter [4] [7] [10] [11]. Recently, new technologies have become available such as medication-impregnated dressings, and of these, chlorhexidineimpregnated gel dressings are the most commonly used [11] [12]. A metanalysis and a systematic review showed that chlorhexidine-impregnated gel dressings prevented CLABSI when compared to other dressings without medication [11] [13]. Chlorhexidine-impregnated gel dressings are changed every seven days or earlier if the integrity of the dressing is compromised, or it becomes soiled or 
wet [7] [14] [15]. Repeated applications and removals of the dressing impact on the skin integrity, causing inflammatory skin reactions and increasing rates of central line-associated bloodstream infection [4] [16] [17]. Although the dressing prevents catheter related infections, there is concern regarding the risk of skin irritation caused by the use of the chlorhexidine-impregnated gel dressings. The most commonly reported adverse effect is contact dermatitis [4] [11] [13]. Studies on chlorhexidine-impregnated gel dressings mainly report the incidence of catheter related infections and do not evaluate the impact of this dressing on the skin as a primary outcome [4] [15] [18] [19] [20]. This study aims to evaluate local cutaneous effects associated with CIGD in patients undergoing HSCT. These patients present extreme immunosuppression due to the treatment they are submitted to, which is aggressive to the skin itself. We believe our results will aid nurses to identify and prevent the development of dressing related skin injuries that may increase the risk of infections to which the patient is already exposed.

\section{Methods}

\subsection{Study Design and Sample}

This was a prospective cross-sectional study conducted at the Bone Marrow Transplantation, Immunotherapy, and Hematology units and at the Outpatient Clinic of a School Hospital in Brazil. Patients with autoimmune or onco-hematological diseases of any age, admitted to the units where the study was conducted, and having a CVC inserted for HSCT were eligible for the study. Exclusion criteria were: patients who had the catheter inserted before hospital admission for HSCT and with a known allergic/hypersensitivity reaction to chlorhexidine. Acute and chronic leukemias, lymphomas, multiple myelomas and bone marrow failures were diagnosed according to World Health Organization (WHO) 2016 criteria [21], the hemoglobinopathies according to the National Institutes of Health (NIH) 2013 Classification of the Disorders of Hemoglobin [22] and systemic sclerosis was according to the American College of Rheumatology/European League Against Rheumatism (ACR/EULAR) 2013 criteria [23].

\subsection{Ethical Considerations}

This study was approved by the Ethics Committee (CAAE-21162213.7.0000.5393). Written informed consent was obtained from adult patients. Patients younger than 18 years old had the family contacted by the investigator who asked for permission to include the patient in the study.

\subsection{Recruitment}

Data were collected from November 2013 to June 2014. Patients were approached by the investigator of the study after receiving CVC and were invited to participate in the study. Inclusion criteria were checked and, if eligible, the study was explained to the participant or his/her family. 


\subsection{Dressing Description}

All included patients received Chlorhexidine-Impregnated Gel Dressings (CIGD) $\left(3 \mathrm{M}^{\mathrm{Tm}}\right.$ Tegaderm $\left.{ }^{\text {Tw }} \mathrm{CHG}\right)$ to cover the catheter exit site while undergoing HSCT. The dressing is composed of a transparent polyurethane film that contains a continuous-release hydrogel with $2 \%$ chlorhexidine (by weight) and a non-woven polyester adhesive tape on the edges [15] [18] [24].

\subsection{Procedure}

Patients who met the inclusion criteria were approached by the researcher after receiving CVC. Non-tunneled CVCs were inserted bedside and tunneled CVCs were surgically inserted, both with maximal sterile barrier precautions. At the beginning of the study, registered nurses responsible for patient care at each participating unit were invited to participate in the study; those who agreed completed a training session to standardize the CIGD application and dressing change procedure. CIGD was applied on the CVC exit site as soon as it stopped bleeding and changed every seven days; unscheduled dressing changes occurred when the dressing was damp, loose, or visibly soiled [7]. Daily, the researcher assessed the patients with CIGD to follow the dressing change procedure performed by registered nurses. The investigator evaluated local cutaneous effects on the skin in contact with the CIGD; assessed through skin evaluation immediately after each dressing removal to detect skin integrity, color abnormalities, or the presence of skin lesions. A retrospective cross-sectional study conducted at the same hospital and care unit in 2011 analyzed patients submitted to allogeneic HSCT that used tunneled catheters and showed that the mean permanence of the catheter was 45 days [25]. Based on this study, we followed the patients for 45 days. If the catheter was removed before this period, data was collected until catheter removal, starting from the day of CVC insertion. Sociodemographic and clinical variables collected were age, length of hospital stay, sex, baseline disease, type of HSCT, chemotherapy conditioning regimens. The catheter variables were type of catheter, insertion vein, catheter time in place, and reasons for catheter removal.

\subsection{Outcomes}

The primary outcome was the occurrence of skin irritation defined as skin loss, erythematous plaques, or vesicles. Secondary outcomes were: -Occurrence of skin erythema which was considered a color abnormality secondary to the mechanical trauma caused by the removal of the dressing; -Discontinuation of the use of the CIGD. In cases that the patient present skin irritation, it was decided if the CIGD should be discontinued or not; -Occurrence of central line-associated bloodstream infection, classified according to the Clinical Practice Guidelines for the Diagnosis and Management of Intravascular Catheter-Related Infection and American Society of Clinical Oncology Clinical Practice Guidelines [9] [26]. In cancer patients, most bloodstream infections are treated without catheter 
withdrawal. The criteria for central line-associated bloodstream infection is differential time to positivity, which is when a blood culture drawn from the catheter hub becomes positive at least two hours earlier than simultaneously drawn peripheral vein blood of equal volume [9] [26]. -Association between skin irritation and the following variables: type of HSCT, type of catheter, catheter time in place and type of chemotherapy conditioning regimens. The type of chemotherapy conditioning regimens were classified according to its intensity: high intensity (Melphalan; association of Bussulfan and Fludarabine; association of Busulfan, Fludarabine and Cyclophosphamide; association of Busulfan, Fludarabine and Immunoglobulin Antithymocyte; association of Busulfan and Cyclophosphamide); medium intensity (association of Carmustine, Etoposide, Cytarabine and Melphalan); and low intensity (Cyclophosphamide; association of Cyclophosphamide and Immunoglobulin Antithymocyte; association of Cyclophosphamide, Immunoglobulin Antithymocyte and Fludarabine; association of Fludarabine, Melphalan and Immunoglobulin Antithymocyte; association of Cyclophosphamide, Fludarabine and total body irradiation) [27].

\subsection{Statistical Analysis}

For statistical analysis, IBM Statistical Package for the Social Sciences (SPSS) software version 24.0 was used. Descriptive simple frequency analysis was developed for nominal and categorical variables. Central trend (mean) and dispersion (standard deviation) measures were developed for continuous variables. The Fisher's exact chi-square test was used to verify possible associations between skin irritation and the following variables: central line-associated bloodstream infection, type of HSCT, type of catheter, catheter time in place, and type of chemotherapy conditioning regimens. Significance was set at $5 \%$.

\section{Results}

A total of 26 patients fulfilled the inclusion criteria. One patient was excluded as the catheter was inserted prior to hospital admission for HSCT. Thus, 25 patients were prospectively evaluated. All patients completed the study follow-up.

Distribution of clinical and sociodemographic characteristics are shown in Table 1.

Most subjects were male 52\% $(\mathrm{n}=13)$ and mean age was 34 years $(\mathrm{SD}=18)$. The mean length of hospital stay was 36 days $(S D=19.4)$. The mean time of the conditioning regimens was 4.8 days $(\mathrm{SD}=1.8)$ and each patient was transplanted only once. Catheter characteristics are shown in Table 2. All patients who underwent allogeneic HSCT $(\mathrm{n}=12)$ had tunneled catheters inserted. Twelve patients who were treated with autologous HSCT received non-tunneled catheters and only one patient submitted to autologous HSCT had a tunneled catheter inserted. Seven of 13 (52\%) patients with tunneled catheters had a remaining catheter for more than 45 days which was the time of the study follow up. According to the local institutional protocol, tunneled catheters can be 
Table 1. Patient clinical and sociodemographic characteristics.

\begin{tabular}{cc}
\hline Characteristics & \\
\hline Age in years, mean (SD*) copy & $34(18.0)$ \\
Length of stay in days, mean (SD) & $36.0(19.4)$ \\
Sex, \% male (n) & $52.0(13)$ \\
Baseline disease, \% (n) & \\
$\quad$ Leukemia $^{\mathrm{a}}$ & $32.0(8)$ \\
Bone marrow failure and hemoglobinopathies & $\mathrm{b}$ \\
Multiple myeloma & $28.0(7)$ \\
$\quad$ Lymphoma & $20.0(5)$ \\
$\quad$ Systemic sclerosis & $12.0(3)$ \\
Type of HSCT, \% (n) & $8.0(2)$ \\
Autologous for onco-hematological disease & \\
Autologous for autoimmune disease & $44.0(11)$ \\
Related allogeneic HLA-matched & $8.0(2)$ \\
Related allogeneic haploidentical & $36.0(9)$ \\
Type of chemotherapy conditioning regimens, \% (n) & $12.0(3)$ \\
$\quad$ Low intensity & \\
Medium intensity & $32.0(8)$ \\
High intensity & $12.0(3)$ \\
\hline
\end{tabular}

${ }^{*} \mathrm{SD}=$ Standard Deviation ${ }^{\mathrm{a}}$ Myeloid or lymphoid acute leukemia, chronic myeloid leukemia; ${ }^{\mathrm{b}}$ sickle cell disease, Fanconi anemia, thalassemia, myelofibrosis, severe aplastic anemia. ${ }^{b}$ conditioning regimen which cannot be classified as medium or high intensity. 'non myeloablative conditioning regimen with minimal cytopenia. ${ }^{\mathrm{d}}$ myeloablative conditioning regimen with irreversible pancytopenia.

Table 2. Overview of catheter characteristics.

\begin{tabular}{|c|c|}
\hline Catheter characteristics & \\
\hline \multicolumn{2}{|l|}{ Type of CVC, \% (n) } \\
\hline Tunneled & $52.0(13)$ \\
\hline Non-tunneled & $48.0(12)$ \\
\hline \multicolumn{2}{|l|}{ Insertion vein, \% (n) } \\
\hline Jugular & $80.0(20)$ \\
\hline Subclavian & $20.0(5)$ \\
\hline \multicolumn{2}{|l|}{ Time in place in days, mean (SD) } \\
\hline Tunneled catheters removed during the study follow up & $27(7.6)$ \\
\hline Non-tunneled catheters & $15(3.6)$ \\
\hline \multicolumn{2}{|l|}{ Reason for tunneled catheter removal, \% (n) } \\
\hline Tunneled catheters that remained more than 45 days & $54.0(7)$ \\
\hline Suspected infection & $23.0(3)$ \\
\hline Diagnosed infection & $8.0(1)$ \\
\hline Catheter was no longer needed & $8.0(1)$ \\
\hline Catheter breakage & $8.0(1)$ \\
\hline \multicolumn{2}{|l|}{ Reason for non-tunneled catheter removal, \% (n) } \\
\hline Hospital discharge & $42.0(5)$ \\
\hline Catheter was no longer needed & $25.0(3)$ \\
\hline Diagnosed infection & $25.0(3)$ \\
\hline Catheter breakage & $8.0(1)$ \\
\hline
\end{tabular}

${ }^{\star} \mathrm{P}$-value from Fisher's exact chi-square test. ${ }^{\mathrm{a}}$ Hematopoietic stem cell transplantation. 
maintained until 100 days after HSCT and non-tunneled catheters must be removed when they are no longer needed or at hospital discharge. Tunneled catheters removed during the study follow up remained for a mean of 27 days $(\mathrm{SD}=7.6)$ and non-tunneled catheters remained for a mean of 15 days $(\mathrm{SD}=$ 3.6). Patients with tunneled catheters had a mean of $5.2(\mathrm{SD}=1.7)$ dressing changes per patient, while in those with non-tunneled catheters the mean was $2.7(\mathrm{SD}=1.1)$. Eleven $(44 \%)$ of 25 patients presented with skin irritation with one or more skin lesions at the same evaluation time point, such as skin loss, erythematous plaques and vesicles. In four (37\%) of these patients we observed skin loss after dressing removal.

Three (27\%) patients presented with skin loss and vesicles, two (18\%) patients presented with skin loss and erythematous plaques and two (18\%) patients presented with skin loss, erythematous plaques and vesicles. Some examples of these skin lesions are shown in Figure 1. Eight (73\%) of eleven patients that presented with skin irritation were submitted to allogeneic HSCT and had a tunneled catheter inserted. Three (27\%) patients who presented with skin irritation were treated with autologous HSCT and received a non-tunneled catheter. The association test between the variables: type of HSCT and skin irritation was statistically significant $(\mathrm{p}=0.03)$. Skin irritation was more frequent in patients who underwent allogeneic HSCT (32\%) and less frequent in autologous HSCT (40\%). Statistical Analysis are shown in Table 3. Considering the three different materials that constituted the CIGD, these lesions were predominantly detected on the skin in contact with the non-woven polyester adhesive tape $(n=22 ; 88 \%)$. The dressings were discontinued in 6 of the 11 patients who presented skin irritation. In two of them, however, it was possible to resume dressing applications, as the injuries were small and easily healed. While the CIGDs were discontinued,

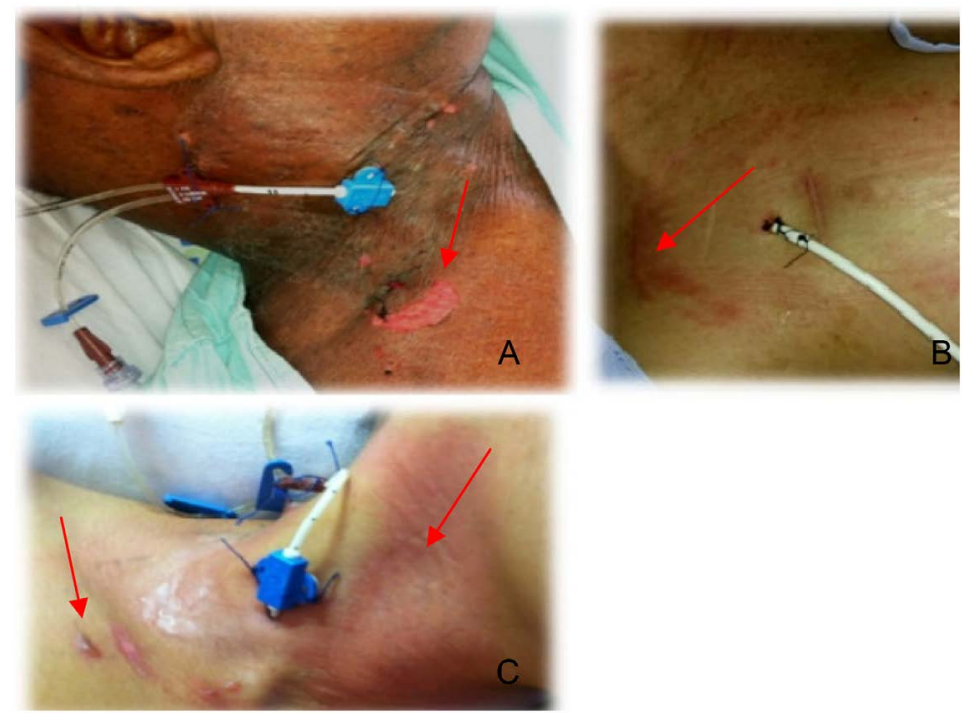

Figure 1. Images of different skin lesions observed in subjects with skin irritation. (A): Areas of skin loss. (B): Erythematous plaque. (C): Presence of vesicle and erythema. 
Table 3. Associations between skin irritation and the following variables: central lineassociated bloodstream infection, type of $\mathrm{HSCT}^{\mathrm{a}}$, catheter time in place, and type of chemotherapy conditioning regimens.

\begin{tabular}{|c|c|c|c|}
\hline \multirow[b]{2}{*}{ Variables } & \multicolumn{3}{|c|}{ Skin irritation } \\
\hline & $\begin{array}{c}\text { Yes } \\
\mathrm{N}=11\end{array}$ & $\begin{array}{c}\text { No } \\
\mathrm{N}=14\end{array}$ & P-value* \\
\hline $\begin{array}{c}\text { Central line-associated } \\
\text { bloodstream infection, \% (n) }\end{array}$ & & & 0.68 \\
\hline $\begin{array}{l}\text { Catheter present infection } \\
\text { occurrence }\end{array}$ & $16.0(4)$ & $16.0(4)$ & \\
\hline Catheter absent infection occurrence & $28.0(7)$ & $40.0(10)$ & \\
\hline Type of HSCT, \% (n) & & & 0.03 \\
\hline Allogenic & $32.0(8)$ & $16.0(4)$ & \\
\hline Autologous & $12.0(3)$ & $40.0(10)$ & \\
\hline Type of catheter, \% (n) & & & 0.67 \\
\hline Tunneled CVC & $32.0(8)$ & $20.0(5)$ & \\
\hline Non-tunneled CVC & $12.0(3)$ & $36.0(9)$ & \\
\hline Catheter time in place, $\%(n)$ & & & 0.67 \\
\hline Less than 20 days & $12.0(3)$ & $36.0(9)$ & \\
\hline More than 20 days & $32.0(8)$ & $20.0(5)$ & \\
\hline $\begin{array}{l}\text { Type of chemotherapy } \\
\text { conditioning regimens, } \%(n)\end{array}$ & & & 0.88 \\
\hline Low intensity & $16.0(4)$ & $16.0(4)$ & \\
\hline Medium intensity & $4.0(1)$ & $8.0(2)$ & \\
\hline High intensity & $24.0(6)$ & $32.0(8)$ & \\
\hline
\end{tabular}

${ }^{\star} \mathrm{P}$-value from Fisher's exact chi-square test. ${ }^{a}$ Hematopoietic stem cell transplantation.

the catheter sites were protected with gauze and adhesive tape or transparent polyurethane film.

Among the 11 patients with skin irritation, three (27\%) developed central line-associated bloodstream infection and the microorganisms found were Corynebacterium sp, carbapenem-resistant Klebsiella pneumonia and Acinetobacter lwoffii. Two patients underwent allogeneic HSCT and one patient underwent autologous HSCT. Conversely, 4 (28\%) of the 14 patients who did not develop skin irritation presented central line-associated bloodstream infection. The microorganisms found were Klebsiella pneumoniae, isolated in two patients, and Comamonas testosterone and Acinetobacter baumannii isolated in the other two patients, respectively. Three subjects underwent autologous and one allogeneic HSCT. The association tests between the variables central line-associated bloodstream infection, type of catheter, catheter time in place, type of chemotherapy conditioning regimens and skin irritation showed no statistical significance. $\mathrm{P}$-values are shown in Table 3. Skin erythema was detected in $100 \%$ of the 25 patients after at least one dressing change procedure. This manifestation was considered a color abnormality secondary to mechanical trauma caused by the 
removal of the dressing. The distribution of the outcomes is shown in Table 4.

\section{Discussion}

The present study evaluated the local cutaneous effects of chlorhexidine-impregnated gel dressings used to cover the central venous catheter exit site of patients undergoing HSCT. Chemotherapy conditioning regimens used in this study didn't have any statistically significant association with skin irritation after CIGD removal. However, studies show that chemotherapy and radiotherapy used in transplant procedures are associated with numerous adverse events affecting skin, mucous membranes, hair, and nails [28]. As examples, melphalan, busulfan, and cyclophosphamide can cause skin or mucosal hyperpigmentation while melphalan is also associated with alopecia and maculopapular rash [28]. A study with children undergoing autologous or allogeneic HSCT showed that approximately $50 \%$ of patients developed some degree of skin damage. Among the different conditioning regimens, this complication was more frequent (80\%) in patients treated with busulfan and thiotepa, presenting as burnlike lesions [29]. Busulfan causes general darkening of the skin secondary to a toxic effect on melanocytes. This effect may be potentiated by other factors, such as the presence of sweat with secreted chemotherapy in areas of skin occluded by adhesive bandages [28]. Skin injured by chemotherapy may strongly adhere to the bandage, being removed along with the dressing characterizing skin loss, which was presented in all the patients of this study who had skin irritation. Skin injuries are usually noticed when adhesive dressings are used for long periods, with numerous dressing change procedures and when the adhesive strength exceeds the skin cell-to-cell interactions leading to dermo-epidermal detachment [29] [30] [31]. Most of our patients with skin irritation and skin loss were submitted to allogeneic HSCT, which usually includes high intensity chemotherapy conditioning regimens. Adhesive dressings have a maximum recommended time in place, according to which changing intervals should be programmed. Additional unscheduled changes may cause skin irritation or worsen effects of radiation and chemotherapy due to damage caused by the adhesive tape [32]. Patients with tunneled catheters were submitted to more dressing change procedures than patients with non-tunneled catheters. This finding was expected due to the time in place of each type of catheter which is longer in tunneled catheters. In addition, skin irritation was more frequent among allogeneic HSCT that used tunneled

Table 4. Overview of the outcomes.

\begin{tabular}{cc}
\hline Outcomes & $\mathrm{N}=\mathbf{2 5}$ \\
\hline Skin irritation, \% (n) & $44.0(11)$ \\
Skin Erythema, \% (n) & $100.0(25)$ \\
Catheter absent infection occurrence & $28.0(7)$ \\
Discontinuation of the CIGD, \% (n) & $24.0(6)$ \\
Central-line associated bloodstream infection, \% (n) & $32.0(8)$ \\
\hline
\end{tabular}


catheters. A randomized clinical trial evaluated children undergoing high dose chemotherapy followed by HSCT and detected a 70\% reduction in the incidence of local skin toxicity when dressings were changed every 15 days instead of every four days without increasing rates of catheter-related infections [29]. Therefore, it has been emphasized that changes scheduled at longer intervals may prevent the onset of skin irritation. On the other hand, extended intervals may increase the rate of unscheduled changes due to detachment or other mechanical events affecting adhesiveness. Nurses and other health professionals should be aware of possible skin irritation when adhesive dressings, including CIGD, are used. CIGD should be maintained for its maximum recommended time in place, which is seven days, avoiding unscheduled changes of the dressing, trying to prevent the onset of skin irritation. However, patients with CIGD should be daily evaluated to detect dressing detachment or mechanical events that require unscheduled dressing change and be aware of higher risk of skin irritation [29] [31]. A multicenter randomized trial [15] carried out in intensive care units, found that of 817 patients who used the CIGD, eight (1\%) presented severe contact dermatitis. This complication was generally observed in severely ill patients with multiple organ failure, subcutaneous edema, and fragile skin. In a different study, these same authors compared different types of transparent dressings in intensive care unit patients and found that skin injury scores were higher in patients using dressings containing chlorhexidine compared to patients using the transparent film without chlorhexidine [19]. A report of seven cases of erosive contact dermatitis due to the CIGD [33] showed that four out of the seven patients were immunosuppressed, and the majority of catheters had been inserted into the femoral vein. Six cases occurred in children from four months to two years of age. In the seven cases described, the dressing was discontinued. This study suggests that children are more susceptible to the irritating effects of dressings with chlorhexidine in their composition. The erosive lesions were attributed to prolonged occlusion of the skin, exposure to components of the dressings, and local concentrations of chlorhexidine. Interestingly, the authors observed a higher incidence of dermatitis on the skin in contact with the chlorhexidine gel. In contrast, in the present study, $88 \%$ of the most severe lesions, classified as skin irritation, were detected predominantly in the areas of contact with the adhesive parts of the dressing. A multicenter randomized trial on chlorhexidine dressings in neutropenic patients detected cutaneous abnormalities in $12.4 \%$ of patients using the dressing. Symptoms observed were erythema, pruritus, vesicles, and skin defects [16]. Thus, in accordance with the literature, our study demonstrates that CIGD is associated with a degree of injury to the skin in contact with it as we also observed some of these symptoms. In our patients, we were able to identify specific predisposing factors, which have been previously described in the literature, such as skin sensitivity due to the toxic effects of chemotherapy, high rate of cell renewal, and use of corticosteroids and secondary immunosuppression to treat the underlying disease, along with che- 
motherapeutic agents in the transplant conditioning regimen. Other factors, such as age, infections, malnutrition, and dehydration may also contribute to the development of skin lesions [31]. Studies on CIGD that evaluated its potential for infection reduction as the first endpoint also evaluated possible skin reactions associated to the dressing. They reported episodes of necrotic lesions, redness, irritation, skin macerations and mild erythema after dressing removal. These lesions are usually self-healing with no need of further intervention [34] [35] [36] [37] [38]. Manifestations of cutaneous effects, such as erythema were observed in $100 \%$ of our patients. However, this manifestation is considered transient and not harmful to the patient, being mostly secondary to the mechanical trauma caused by the removal of the dressing [31] [35]. In our patients, we did not observe an impact of CIGD-derived skin injuries on the incidence of bloodstream infections, whether catheter-related or not. Similar studies have also not confirmed this association [11] [15] [16] [19] [33]. In fact, in the report of seven cases of erosive contact dermatitis due to the CIGD, all cultures and microbiological evaluations from the sites presented negative results [33]. A large study that compared chlorhexidine-containing and non-containing CVC dressings demonstrated a significant reduction in local contamination in patients using the chlorhexidine-impregnated dressings. However, the authors were not able to correlate these findings with catheter-related infections [35]. The limitations of the present study were that we were not always able to identify which lesions were directly due to the dressings and which were associated with other local or systemic factors, such as allergic rashes, graft versus host disease, and viral infections, among other conditions. The majority of HSCT studies have small sample sizes due to the restricted number of transplants. Therefore, a large sample is only possible in a multicenter trial. Further studies, under more controlled conditions, with a larger number of participants, are still warranted. Nevertheless, our findings are clinically relevant and should contribute to patient assistance and health care professional education.

\section{Conclusion}

In this study, we detected that skin irritation was more frequent in patients who underwent allogeneic HSCT. The most common skin manifestation was skin loss. Lesions such as skin loss, erythematous plaques, or vesicles were classified as skin irritation, potentially leading to discontinuation of the dressing. We also observed that most skin irritation events occurred in areas in contact with the adhesive bandage of the dressing. This study indicates that health professionals should carefully evaluate each patient individually for use of CIGD, considering the risk of local lesions and ideal changing intervals.

\section{References}

[1] Ljungman, P., Urbano-Ispizua, A., Cavazzana-Calvo, M., Demirer, T., Dini, G., Einsele, H., Gratwohl, A., Madrigal, A., Niederwieser, D., Passweg, J., Rocha, V., 
Saccardi, R., Schouten, H., Schmitz, N., Socie, G., Sureda, A. and Apperley, J. (2006) Allogeneic and Autologous Transplantation for Haematological Diseases, Solid Tumours and Immune Disorders: Definitions and Current Practice in Europe. Bone Marrow Transplantation, 37, 439-449. https://doi.org/10.1038/sj.bmt.1705265

[2] Ljungman, P., Bregni, M., Brune, M., Cornelissen, J., de Witte, T., Dini, G., Einsele, H., Gaspar, H.B., Gratwohl, A., Passweg, J., Peters, C., Rocha, V., Saccardi, R., Schouten, H., Sureda, A., Tichelli, A., Velardi, A. and Niederwieser, D. (2010) Allogeneic and Autologous Transplantation for Haematological Diseases, Solid Tumours and Immune Disorders: Current Practice in Europe 2009. Bone Marrow Transplantion, 45, 219-234. https://doi.org/10.1038/bmt.2009.141

[3] Pasquini, M.C. and Zhu, X. (2014) Current Uses and Outcomes of Hematopoietic Stem Cell Transplantation: 2014 CIBMTR Summary Slides. http://www.cibmtr.org

[4] Gavin, N.C., Webster, J., Chan, R.J. and Rickard, C.M. (2016) Frequency of Dressing Changes for Central Venous Access Devices on Catheter-Related Infections. Cochrane Database Systematic Review, 2, 1-52.

[5] Centers for Diseases Control and Prevention (CDC) (2018) Bloodstream Infection Event (Central Line-Associated Bloodstream Infection and Non-Central Line Associated Bloodstream Infection).

https://www.cdc.gov/nhsn/pdfs/pscmanual/4psc_clabscurrent.pdf

[6] Webster, J., Gillies, D., Riordan, O.E., Kl, S., Cm, R., Webster, J., Gillies, D., Riordan, E.O., Sherriff, K.L. and Rickard, C.M. (2011) Gauze and Tape and Transparent Polyurethane Dressings for Central Venous Catheters. The Cochrane Library, 11, 2011-2013. https://doi.org/10.1002/14651858.CD003827.pub2

[7] O’Grady, N.P., Alexander, M., Burns, L.A., Dellinger, E.P., Garland, J., Heard, S.O., Lipsett, P.A., Masur, H., Mermel, L.A., Pearson, M.L., Raad, I.I., Randolph, A.G., Rupp, M.E. and Saint, S. (2011) Guidelines for the Prevention of Intravascular Catheter-Related Infections. Clinical Infectious Diseases, 52, e162-e193.

[8] Safdar, N. and Maki, D.G. (2004) The Pathogenesis of Catheter-Related Bloodstream Infection with Noncuffed Short-Term Central Venous Catheters. Intensive Care Medicine, 30, 62-67. https://doi.org/10.1007/s00134-003-2045-z

[9] Mermel, L.A., Allon, M., Bouza, E., Craven, D.E., Flynn, P., O’Grady, N.P., Raad, I.I., Rijnders, B.J.A., Sherertz, R.J. and Warren, D.K. (2009) Clinical Practice Guidelines for the Diagnosis and Management of Intravascular Catheter-Related Infection: 2009 Update by the Infectious Diseases Society of America. Clinical Infectious Diseases, 49, 1-45. https://doi.org/10.1086/599376

[10] Rippon, M., White, R. and Davies, P. (2007) Skin Adhesives and Their Role in Wound Dressings. Wounds UK, 3, 76-86.

[11] Ullman, A.J., Cooke, M.L., Mitchell, M., Lin, F., New, K., Long, D.A., Mihala, G. and Rickard, C.M. (2015) Dressings and Securement Devices for Central Venous Catheters (CVC). Cochrane Database of Systematic Reviews, No. 9, CD010367.

[12] Arvaniti, K., Lathyris, D., Clouva-Molyvdas, P., Haidich, A.B., Mouloudi, E., Synnefaki, E., Koulourida, V., Georgopoulos, D., Gerogianni, N., Nakos, G. and Matamis, D. (2012) Comparison of Oligon Catheters and Chlorhexidine-Impregnated Sponges with Standard Multilumen Central Venous Catheters for Prevention of Associated Colonization and Infections in Intensive Care Unit Patients: A Multicenter, Randomized, Controlled Study. Critical Care Medicine, 40, 420-429. https://doi.org/10.1097/CCM.0b013e31822f0d4b

[13] Safdar, N., O’Horo, J.C., Ghufran, A., Bearden, A., Didier, M.E., Chateau, D. and Maki, D.G. (2014) Chlorhexidine-Impregnated Dressing for Prevention of Catheter- 
Related Bloodstream Infection: A Metaanalysis. Critical Care Medicine, 42, 1703 1713. https://doi.org/10.1097/CCM.0000000000000319

[14] Loveday, H.P., Wilson, J.A., Pratt, R.J., Golsorkhi, M., Tingle, A., Bak, A., Browne, J., Prieto, J. and Wilcox, M. (2014) Epic3: National Evidence-Based Guidelines for Preventing Healthcare-Associated Infections in NHS Hospitals in England. Journal of Hospital Infection, 86, S1-S70. https://doi.org/10.1016/j.jhin.2013.09.010

[15] Timsit, J.F., Schwebel, C., Bouadma, L., Geffroy, A., Garrouste-Orgeas, M., Pease, S., Herault, M.-C., Haouache, H., Calvino-Gunther, S., Gestin, B., Armand-Lefevre, L., Leflon, V., Chaplain, C., Benali, A., Francais, A., Adrie, C., Zahar, J.R., Thuong, M., Arrault, X., Croize, J. and Lucet, J.C. (2009) Chlorhexidine-Impregnated Sponges and Less Frequent Dressing Changes for Prevention of Catheter-Related Infections in Critically Ill Adults: A Randomized Controlled Trial. JAMA, 301, 1231-1241. https://doi.org/10.1001/jama.2009.376

[16] Biehl, L.M., Huth, A., Panse, J., Krämer, C., Hentrich, M., Engelhardt, M., Ko, G., Kiehl, M., Wendtner, C., Karthaus, M., Ullmann, A.J., Hellmich, M., Christ, H. and Vehreschild, M.J.G.T. (2016) A Randomized Trial on Chlorhexidine Dressings for the Prevention of Catheter-Related Bloodstream Infections in Neutropenic Patients. Annals of Oncology, 27, 1916-1922. https://doi.org/10.1093/annonc/mdw275

[17] Cutting, K.F. (2008) Impact of Adhesive Surgical Tape and Wound Dressings on the Skin, with Reference to Skin Stripping. Journal of Wound Care, 17, 157-162. https://doi.org/10.12968/jowc.2008.17.4.28836

[18] Ruschulte, H., Franke, M., Gastmeier, P., Zenz, S., Mahr, K.H., Buchholz, S., Hertenstein, B., Hecker, H. and Piepenbrock, S. (2009) Prevention of Central Venous Catheter Related Infections with Chlorhexidine Gluconate Impregnated Wound Dressings: A Randomized Controlled Trial. Annals of Hematology, 88, 267-272. https://doi.org/10.1007/s00277-008-0568-7

[19] Timsit, J.F., Bouadma, L., Ruckly, S., Schwebel, C., Garrouste-Orgeas, M., Bronchard, R., CalvinoGunther, S., Laupland, K., Adrie, C., Thuong, M., Herault, M.C., Pease, S., Arrault, X. and Lucet, J.C. (2012a) Dressing Disruption Is a Major Risk Factor for Catheter-Related Infections. Critical Care Medicine, 40, 1707-1714.

[20] Timsit, J.F., Mimoz, O., Mourvillier, B., Souweine, B., Garrouste-Orgeas, M., Alfandari, S., Plantefeve, G., Bronchard, R., Troche, G., Gauzit, R., Antona, M., Canet, E., Bohe, J., Lepape, A., Vesin, A., Arrault, X., Schwebel, C., Adrie, C., Zahar, J.R., Ruckly, S., Tournegros, C. and Lucet, J.C. (2012b) Randomized Controlled Trial of Chlorhexidine Dressing and Highly Adhesive Dressing for Preventing CatheterRelated Infections in Critically Ill Adults. American Journal of Respiratory and Critical Care Medicine, 186, 1272-1278.

[21] Arber, D.A., Orazi, A., Hasserjian, R., Thiele, J., Borowitz, M.J., Le Beau, M.M., Bloomfield, C.D., Cazzola, M. and Vardiman, J.W. (2016) The 2016 Revision to the World Health Organization Classification of Myeloid Neoplasms and Acute Leukemia. Blood, 127, 2391-2405. https://doi.org/10.1182/blood-2016-03-643544

[22] Forget, B.G. and Bunn, H.F. (2013) Classification of the Disorders of Hemoglobin. Cold Spring Harbor Perspectives in Medicine, 3, a011684. https://doi.org/10.1101/cshperspect.a011684

[23] van den Hoogen, F., Khanna, D., Fransen, J., Johnson, S.R., Baron, M., Tyndall, A., Matucci-Cerinic, M., Naden, R.P., Medsger, T.A. Jr., Carreira, P.E., Riemekasten, G., Clements, P.J., Denton, C.P., Distler, O., Allanore, Y., Furst, D.E., Gabrielli, A., Mayes, M.D., van Laar, J.M., Seibold, J.R., Czirjak, L., Steen, V.D., Inanc, M., Kowal-Bielecka, O., Müller-Ladner, U., Valentini, G., Veale, D.J., Vonk, M.C., Walker, U.A., Chung, L., Collier, D.H., Csuka, M.E., Fessler, B.J., Guiducci, S., Herrick, A., 
Hsu, V.M., Jimenez, S., Kahaleh, B., Merkel, P.A., Sierakowski, S., Silver, R.M., Simms, R.W., Varga, J. and Pope, J.E. (2013) Classificationcriteria for Systemic Sclerosis: An ACR-EULAR Collaborative Initiative. Arthritis and Rheumatism, 65, 2737-2747.

[24] Bashir, M.H., Olson, L.K.M. and Walters, S.A. (2012) Suppression of Regrowth of Normal Skin Flora under Chlorhexidine Gluconate Dressings Applied to Chlorhexidine Gluconate-Prepped Skin. American Journal of Infection Control, 40, 344-348. https://doi.org/10.1016/j.ajic.2011.03.030

[25] Castanho, L.C., Silveira, R.C.C.P., Braga, F.T.M.M., Canini, S.R.M.S., Reis, P.E.D. and Voltarelli, J.C. (2011) Motivo de retirada do cateter de Hickman em pacientes submetidos ao transplante de células-tronco hematopoéticas. Acta Paulista de Enfermagem, 24, 244-248. https://doi.org/10.1590/S0103-21002011000200014

[26] Schiffer, C.A., Mangu, P.B., Wade, J.C., Camp-Sorrell, D., Cope, D.G., El-Rayes, B.F., Gorman, M., Ligibel, J., Mansfield, P. and Levine, M. (2013) Central Venous Catheter Care for the Patient with Cancer: American Society of Clinical Oncology Clinical Practice Guideline. Journal of Clinical Oncology, 31, 1357-1370. https://doi.org/10.1200/JCO.2012.45.5733

[27] Bacigalupo, A., Ballen, K., Rizzo, D., Giralt, S., Lazarus, H., Ho, V., Apperley, J., Slavin, S., Pasquini, M., Sandmaier, B.M., Barrett, J., Blaise, D., Lowski, R. and Horowitz, M. (2009) Defining the Intensity of Conditioning Regimens: Working Definitions. Biology of Blood and Marrow Transplantation, 15, 1628-1633. https://doi.org/10.1016/j.bbmt.2009.07.004

[28] Payne, A.S. and Savarese, D.M. (2016) Cutaneous Complications of Conventional Chemotherapy Agents.

https://www.uptodate.com/contents/cutaneous-complications-of-conventional-che motherapyagents

[29] Benhamou, E., Fessard, E., Com-Nougue, C., Beaussier, P.S., Nitenberg, G., Tancrede, C., Dodeman, S. and Hartmann, O. (2002) Less Frequent Catheter Dressing Changes Decrease Local Cutaneous Toxicity of High-Dose Chemotherapy in Children, without Increasing the Rate of Catheter-Related Infections: Results of a Randomised Trial. Bone Marrow Transplantation, 29, 653-658.

https://doi.org/10.1038/sj.bmt.1703511

[30] Gao, Y., Wang, X., Chen, S., Li, S. and Liu, X. (2013) Acute Skin Barrier Disruption with Repeated Tape Stripping: An in Vivo Model for Damage Skin Barrier. Skin Research and Technology, 19, 162-168. https://doi.org/10.1111/srt.12028

[31] McNichol, L., Lund, C., Rosen, T. and Gray, M. (2013) Medical Adhesives and Patient Safety: State of the Science: Consensus Statements for the Assessment, Prevention, and Treatment of Adhesive-Related Skin Injuries. Orthopedic Nursing, 32, 267-281. https://doi.org/10.1097/NOR.0b013e3182a39caf

[32] Rasero, L., Degl'Innocenti, M., Mocali, M., Alberani, F., Boschi, S., Giraudi, A., Arnaud, M.T., Zucchinali, R., Paris, M.G., Dallara, R., Thaler, S., Perobelli, G., Parfazi, S., De Lazzer, T. and Peron, G. (2000) Comparison of Two Different Time Interval Protocols for Central Venous Catheter Dressing in Bone Marrow Transplant Patients: Results of a Randomized, Multicenter Study. The Italian Nurse Bone Marrow Transplant Group (GITMO). Haematologica, 85, 275-279.

[33] Weitz, N., Lauren, C.T., Weiser, J., LeBoeuf, N.R., Grossman, M.E., Biagas, K., Garzon, M.C. and Morel, K.D. (2013) Chlorhexidine Gluconate-Impregnated Central Access Catheter Dressings as a Cause of Erosive Contact Dermatitis: A Report of 7 Cases. JAMA Dermatology, 149, 195-199.

https://doi.org/10.1001/jamadermatol.2013.903 
[34] Thokala, P., Arrowsmith, M., Poku, E., Martyn-St, M., James, A.J., Foster, S., Elliott, T. and Whitehouse, T. (2016) Economic Impact of Tegaderm Chlorhexidine Gluconate (CHG) Dressing in Critically Ill Patients. Journal of Infection Prevention, 17, 216-223. https://doi.org/10.1177/1757177416657162

[35] Karpanen, T.J., Casey, A.L., Whitehouse, T., Nightingale, P., Das, I. and Elliott, T.S.J. (2016) Clinical Evaluation of a Chlorhexidine Intravascular Catheter Gel Dressing on Short-Term Central Venous Catheters. American Journal of Infection Control, 44, 54-60. https://doi.org/10.1016/j.ajic.2015.08.022

[36] Scheithauer, S., Lewalter, K., Schroder, J., Koch, A., Hafner, H., Krizanovic, V., Nowicki, K., Hilgers, R.D. and Lemmen, S.W. (2014) Reduction of Central Venous Line-Associated Bloodstream Infection Rates by Using a Chlorhexidine-Containing Dressing. Infection, 42, 155-159. https://doi.org/10.1007/s15010-013-0519-7

[37] Wall, J.B., Divito, S.J. and Talbot, S.G. (2014) Chlorhexidine Gluconate-Impregnated Central-Line Dressings and Necrosis in Complicated Skin Disorder Patients. Journal of Critical Care, 29, 1130.e1-1130.e4.

[38] Jenks, M., Craig, J., Green, W., Hewitt, N., Arber, M. and Sims, A. (2016) Tegaderm CHG IV Securement Dressing for Central Venous and Arterial Catheter Insertion Sites: A Nice Medical Technology Guidance. Applied Health Economics and Health Policy, 14, 135-149. https://doi.org/10.1007/s40258-015-0202-5 